
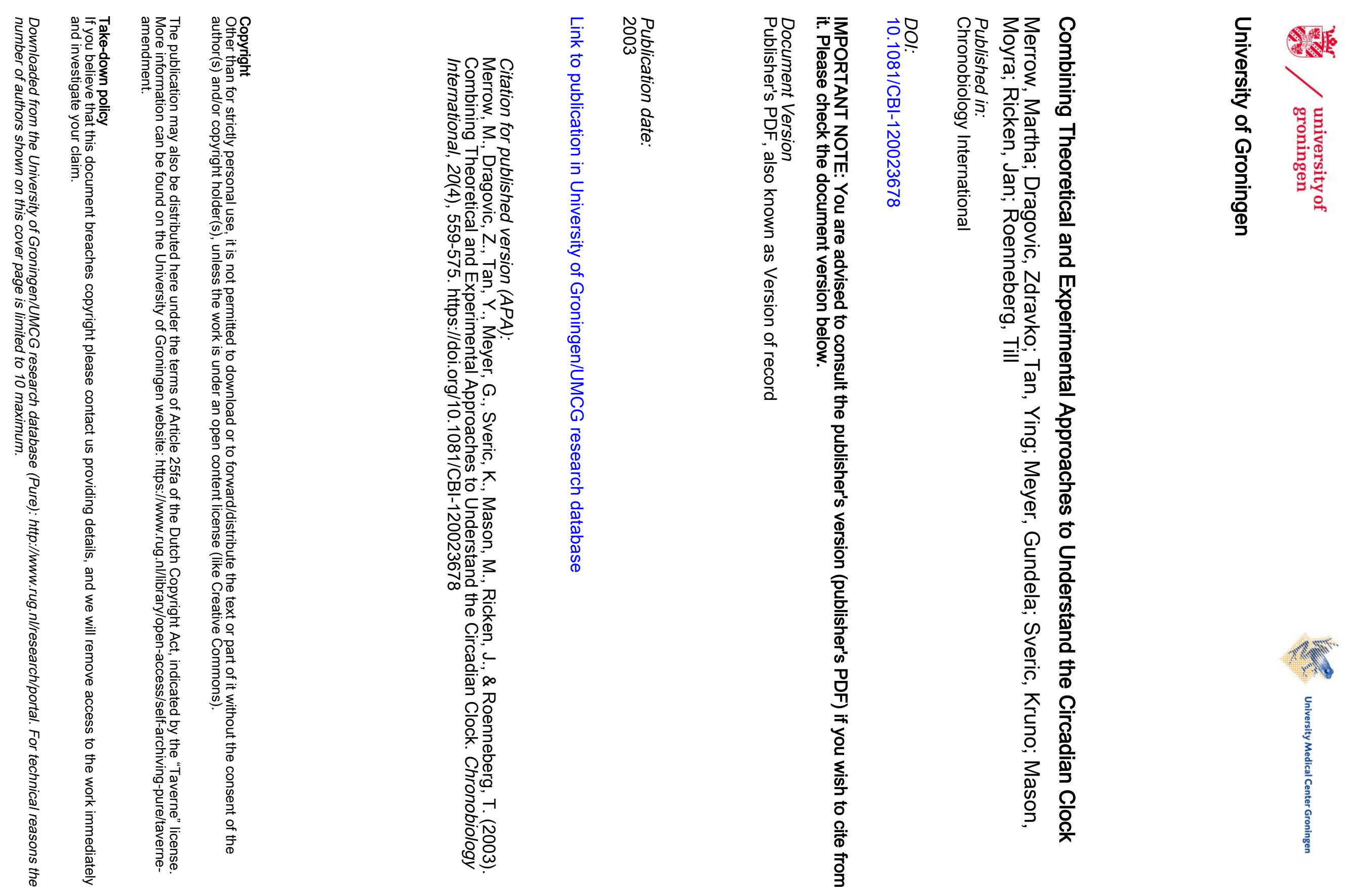


\title{
ORDER
}

CHRONOBIOLOGY INTERNATIONAL

Vol. 20, No. 4, pp. 559-575, 2003

REVIEW

\section{Combining Theoretical and Experimental Approaches to Understand the Circadian Clock}

\author{
Martha Merrow, ${ }^{1, *}$ Zdravko Dragovic, ${ }^{1}$ Ying Tan, ${ }^{1}$ Gundela Meyer, ${ }^{1}$ \\ Kruno Sveric, ${ }^{1}$ Moyra Mason, ${ }^{1,2}$ Jan Ricken, ${ }^{1}$ and Till Roenneberg ${ }^{1}$ \\ ${ }^{1}$ Institut für Medizinische Psychologie, Munich, Germany \\ ${ }^{2}$ Dipartimento di Biologia, University of Padova, Padova, Italy
}

\begin{abstract}
This review is intended as a summary of our work carried out as part of the German Research Association (DFG) Center Program on Circadian Rhythms. Over the last six years, our approach to understanding circadian systems combined theoretical and experimental tools, and Gonyaulax and Neurospora have proven ideal for these efforts. Both of these model organisms demonstrate that even simple circadian systems can have multiple light input pathways and more than one rhythm generator. They have both been used to elaborate basic circadian features in conjunction with formal models. The models introduce the "zeitnehmer," i.e., a clock-regulated input pathway, to the conceptual framework of circadian systems, and proposes networks of individual feedbacks as the basis for circadian rhythmicity.
\end{abstract}

Key Words: Neurospora; Gonyaulax; Zeitnehmer; Feedback entrain; Light.

\section{INTRODUCTION: A BASIS FOR COMPLEXITY}

Circa $24 \mathrm{~h}$ oscillations that continue in the absence of external stimuli have been described in organisms from prokaryotes to man. These circadian rhythms are manifest at many levels, from behavior and physiology to gene expression. Although a key

${ }^{*}$ Correspondence: Martha Merrow, Institut für Medizinische Psychologie, Goethestrasse 31, Munich, Germany; E-mail: martha.merrow@imp.med.uni-muenchen.de. 
experimental observation in the laboratory is self-sustained rhythmicity under constant conditions, circadian systems are almost exclusively entrained or synchronized with the environment in nature. An important relationship between free-running rhythms and the entrained circadian system is the "phase of entrainment" (Roenneberg et al., 2003). The phase refers to the time of day at which a discernable event occurs within the cycle (e.g., for birds, a marked increase in locomotor activity commences in anticipation of dawn). Entrainment refers to a stable phase relationship between biological rhythms and physical oscillations (e.g., a light cycle). Although we know that circadian systems will be more complex than mechanical oscillators, some features of the former are highly similar to the behavior of the latter (e.g., phase shifting or resetting behavior). As for a mechanical oscillator, the longer the free-running period $(\tau)$ of a biological oscillator, the later within the day will the rhythm entrain, and vice versa: earlier entrainment occurs with shorter free-running periods (hence the connection between $\tau$ and phase of entrainment). The reciprocal effect is seen when organisms with the same $\tau$ are put into longer or shorter entraining cycles (called T cycles). In shorter Ts, the entrained phase is later than in long ones (Aschoff, 1979). So, investigations into what determines the length of $\tau$ and how it is influenced by environmental factors also pertain to entrainment. Early insights into molecular aspects of circadian rhythmicity were based on extensive physiological experimentation. More recently, genetic approaches have been relied on for determining clock components. In our work on simple circadian model systems, the unicellular alga Gonyaulax and the filamentous fungus Neurospora, we have found the combination of physiology and genetics to be a powerful tool.

An initial step to unravel a biological system is to conceptualize and model it. Any circadian system will have an input pathway to transduce information from the exogenous environment to the endogenous clock. The external signals that are capable of entraining circadian systems are called zeitgebers (German for "time giver"). Zeitgebers include light, temperature, food, and social cues. Input pathways feed into a rhythm generator, responsible for the generation of the circa $24 \mathrm{~h}$ oscillation. The last building blocks of circadian systems are output pathways, driving the rhythmic phenotypes.

The physiological and ecological description of the unicellular alga, Gonyaulax polyedra, has contributed greatly to our understanding of circadian programs, in general. The algae have numerous clock-controlled outputs, some of which can be monitored simultaneously. Two forms of bioluminescent expression can be recorded: a sustained "glow" at the end of the night, and much brighter, short flashes occurring throughout the night. The adaptive function of the bioluminescence has never been experimentally demonstrated, but is thought to deter predator attacks by copepods (Buskey and Mills, 1983). Other rhythmic outputs are found in, or are facilitated by, the algae's migration behavior. During the day, the algae rise to the surface, where they harvest light for photosynthesis. At night they scavenge nitrogen from the depths; thus, energy, oxygen, carbon, and nitrogen sources are facilitated by the circadian program. At the ocean's surface, Gonyaulax populations form aggregations. With the combination of a camera and a photomultiplier tube, both bioluminescence and aggregation can be recorded and quantified. When the algae are held in constant white light with sufficient nitrogen, all circadian outputs maintain a stable phase relationship with one another-they, therefore, have the same $\tau$. When cultures are incubated in constant red light or deprived of nitrogen, the rhythms of bioluminescence and aggregation can be dissociated, and are sometimes observed as free-running with different (circadian) $\tau$ 's (Roenneberg and Morse, 1993; 


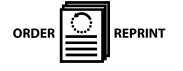

Combining Theory and Experiment to Understand Circadian Clock

Roenneberg and Rehman, 1996). Thus, at least two circadian subsystems contribute to the temporal program of this unicellular organism.

Another contribution from the Gonyaulax work is the demonstration of clockregulated light input pathways. Using spectrally defined light and pharmacological agents, blue and blue-red sensitive light transduction pathways to the circadian system can be distinguished (Roenneberg and Hastings, 1988; Roenneberg and Deng, 1997). The blue input pathway is active only during the subjective night, facilitating large (type 0 ) phase shifts, while the blue-red input is active throughout the circadian cycle and leads to small (type 1) phase shifts. Thus the responsiveness and the spectral sensitivity of the light input pathways are under clock control. Using other model organisms, clock regulation of light input pathways has also been clearly shown (Bognar et al., 1999; Fleissner and Fleissner, 1996; Somers et al., 1998). In retrospect, given the extensive regulation of cellular physiology by the circadian clock, this observation is not surprising.

How do these physiological experiments contribute to an understanding of the molecular mechanism of circadian rhythms? While several of the properties and responses of circadian systems resemble those of simple mechanical oscillators, probing the Gonyaulax circadian system reveals a multioscillator and multi-input construction.
\end{abstract}

\title{
MODELING: COMBINING TWO FEEDBACK LOOPS
}

When considered together, two oscillators in a single cell and clock-regulated light input pathways are conceptually problematic. In any oscillatory system, the inputs have profound effects on phase, period, and amplitude (Glass and Mackey, 1988). Thus, alterations (e.g., a mutation) of an endogenously oscillating input pathway could have effects indistinguishable from alterations of a central component of the rhythm generator.

The problem becomes more acute in view of the molecular genetic description of the circadian clock. Mutant hunts have delivered clock genes in flies, fungi, animals, plants, and cyanobacteria. Due to the loss of clock function in clock mutant strains, these genes are generally considered to produce central components of the rhythm generation mechanism. Expression patterns of the first clock gene, dPeriod, crystallized the transcription/translation negative feedback loop hypothesis, whereby the protein product feeds back negatively—directly or indirectly—on its own transcription (Hardin et al., 1990). A circadian negative feedback was also experimentally probed in Neurospora using the clock gene frequency ( $f r q$ ). Induced overexpression of the gene results in loss of overt circadian rhythms as well as a decrease in RNA expression (Aronson et al., 1994b). If these clock genes function either on input pathways or within the rhythm generator, they could similarly impact circadian rhythms in mutant strains or overexpression experiments, and it would be difficult to distinguish between these possibilities.

To assess the potential of a clock gene functioning on an input pathway to a rhythm generator, we modeled an "input feedback" (Roenneberg and Merrow, 1998) based on the following assumptions: (a) feedbacks are commonly found in biological pathways (controlling responsiveness, homeostasis, or adaptation) and (b) light input pathways are commonly clock-regulated (Bognar et al., 1999; Fleissner and Fleissner, 1987). The model essentially consists of two mutually coupled feedback loops (Fig. 1). Both the feedback within the input pathway and the downstream oscillator are simple, two-component 

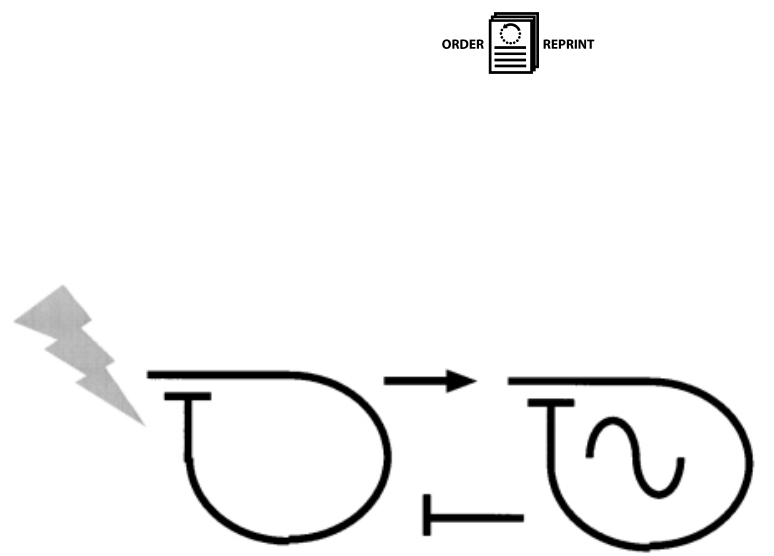

Figure 1. The input feedback model. The rhythm generator (feedback loop on right) is downstream of a light input pathway that is itself clock regulated. The zeitgeber (indicated as a lightning bolt) need not be light, but could also be nitrate, in the case of Gonyaulax (Roenneberg and Rehman, 1996).

negative feedback loops (e.g., RNA and protein of a clock gene). The parameters of the model were set so that the isolated oscillator loop is self-sustained, while the isolated input loop damps. When the components are put together, the system produces a self-sustained rhythm.

The model was used to investigate what happens to circadian qualities when the input feedback is compromised or removed. The results show that most of the properties found in clock mutants can be simulated by changing the properties of the input feedback (Roenneberg and Merrow, 1998). Namely:

1. The input feedback has a profound effect on $\tau$ and can even turn the self-sustained oscillation into a damped rhythm, eventually leading to arhythmicity.

2. Constitutive overexpression of the input component stops the rhythm.

3. Temperature compensation (different temperatures were simulated by assigning temperature-dependent rates to the different equations) is lost when the input loop is uncoupled from the oscillator (see Rensing et al., 1997; Ruoff and Rensing 1996; Ruoff et al., 1996; 1997 for further mathematical exploration of temperature-compensated circadian systems).

4. Finally, the phase response curve (PRC) based on light pulses (simulated by increasing the level of the input at discrete times) depends on the properties of the input feedback.

\section{EXPERIMENTS (1): THE POWER OF ENTRAINMENT}

How can theoretical models be used practically to gain insights into real circadian systems? There are indications that the two oscillators in Gonyaulax are targets of one or the other light input pathway (Morse et al., 1994; Roenneberg and Deng, 1997). To investigate these possibilities further, the molecular mechanisms of the two oscillators as well as the identity of the photopigments and their transduction pathways have to be elucidated. Unfortunately, Gonyaulax does not lend itself to molecular genetic experimentation [its scrambled genome is about 50 times larger than the human genome (Lee et al., 1993), and at this time it is not easily transformable], so it is a poor choice for further work on the molecular mechanism of the circadian system. In contrast, Neurospora is 
amenable to forward and reverse genetics, and several components of the clock mechanism as well as of the light input pathway have been cloned. It has a small, (recently) sequenced genome and is haploid, so that even recessive mutations are detectable without backcrossing. In addition, Neurospora is easy to grow, nonpathogenic, and inexpensive to maintain. Over the last three decades, $N$. crassa has been among the premier molecular genetic systems for both clock and photobiology research.

Using the readout of a $22 \mathrm{~h}$ free-running rhythm in asexual spore formation, Neurospora was used to generate a panel of clock mutants (Feldman and Hoyle, 1973). The frq gene was the first to be cloned (McClung et al., 1989) and, over the last $10 \mathrm{yrs,} \mathrm{a}$ molecular, coregulatory, clock network has been described. On the oscillator side, frq transcription is positively regulated by WHITE COLLAR-1 (WC-1) and WHITE COLLAR-2 (WC-2) (Crosthwaite et al., 1997), and the FRQ protein feeds back negatively on its own transcription (Aronson et al., 1994). Furthermore, FRQ has positive effects on $w c-1$ transcription (Merrow et al., 2001b), and on WC-1, and WC-2 protein levels (Cheng et al., 2001; Lee et al., 2000); finally, WC-1 has negative effects on $w c-2$ RNA (Cheng et al., 2002). On the light input side, $f r q$ and $w c-1$ are rapidly induced by light (Ballario et al., 1996; Crosthwaite et al., 1995), and WC-1 and WC-2 are phosphorylated (Schwerdtfeger and Linden, 2000; Talora et al., 1999) in a light-dependent manner. Both WC proteins contain PAS domains, and a third Neurospora PAS protein, VIVID (VVD) also has a role in circadian light signaling, e.g., on release from light to darkness, conidiation is delayed by about $4 \mathrm{~h}$ in some - though not all — vvd mutants (Heintzen et al., 2001; Shrode et al., 2001). A full-length, antisense frq transcript also regulates the timing of conidiation (Kramer et al., 2003). Finally, the WC-1 protein was recently demonstrated to function as a blue-light photoreceptor (Froehlich et al., 2002; He et al., 2002), and in addition, there are a number of kinases that are critical for stability of the FRQ protein and thus the circadian, free-running period (Görl et al., 2001; Liu et al., 2000; Yang et al., 2002).

The convergence of light input and clock components makes Neurospora an ideal model organism for testing the hypothesis of clock genes as part of an input feedback. A simple prediction of the model described above is that circadian qualities remain when the input feedback is removed, for example, by mutation of one of its genes (Roenneberg and Merrow, 1998). In such a restricted system, the circadian free-running rhythms may not be self-sustained, and entrainment by light may be lost. Remaining qualities of the circadian system could, however, be uncovered by using an alternative input pathway for entrainment, e.g., temperature. As described in the introduction, the characteristics of circadian entrainment can be experimentally demonstrated in zeitgeber cycles of different lengths ( $\mathrm{T}$ cycles). With shorter T cycles, the phase of entrainment settles to a later time relative to the zeitgeber transitions (Aschoff and Pohl, 1978). At a certain point (at the limit of entrainment), the cycle becomes too short to entrain the system, which then free-runs through the zeitgeber cycle with relative coordination (Holst, 1939). When $\mathrm{T}$ becomes even shorter $(\mathrm{T} \approx \tau / 2)$, the entrained output is seen once per two cycles, called a frequency demultiplication (Bruce, 1960). These characteristics of entrainment offer an excellent benchmark to probe for residual circadian properties in arrhythmic clock mutants. We, therefore, studied the entrainment of different frq mutants in Neurospora by light and temperature cycles of different lengths.

In temperature cycles, the lab Neurospora strain (band) follows the rules of circadian entrainment (Merrow et al., 1999). The entrained phase changes systematically with T, moving progressively later as the $\mathrm{T}$ shortens [Fig. 2(A)]. Frequency demultiplication 
occurs in $12 \mathrm{~h}$ cycles (e.g., one conidial band per $24 \mathrm{~h}$ ), and finally, the phase of entrainment is a function of zeitgeber strength.

Surprisingly, in light/dark (LD) cycles, none of these prototypical circadian entrainment characteristics are apparent in Neurospora (Merrow et al., 1999): the onset of conidiation is pegged to the $\mathrm{L} \rightarrow \mathrm{D}$ transition, occurring 7-8h later, regardless of the length of T [Fig. 2(B)]. "Fixed" phases of entrainment were also found for the long and short period mutants ( $f r q^{7}$ and $f r q^{1}$, respectively), and the delay after the $\mathrm{L} \rightarrow \mathrm{D}$ transition was a function of the respective mutant's $\tau$ : relative to $\mathrm{frq}^{+}(\tau \approx 22 \mathrm{~h})$, onset of conidiation was earlier for $f r q^{1}(\tau \approx 16 \mathrm{~h})$ and later for $f r q^{7}(\tau \approx 29 \mathrm{~h})$. The fixed phases were, in contrast to the results from temperature cycles, insensitive to different zeitgeber strengths, i.e., they did not change until a threshold was reached at low fluences below which the rhythms ran free. These results indicate a driven, noncircadian light-resetting behavior. There are, however, two observations indicating that synchronization by light in Neurospora may still be achieved via circadian entrainment: (a) After transition from constant darkness to LD conditions, transients of several days can occur until a stable, entrained state is reached (a driven response would stabilize in the first or second cycle) and (b) In T cycles close to $24 \mathrm{~h}$, the phase for the wild type strain was advanced by one to two hours relative to shorter cycles [see ski-shaped line in Fig. 2(B)].

A

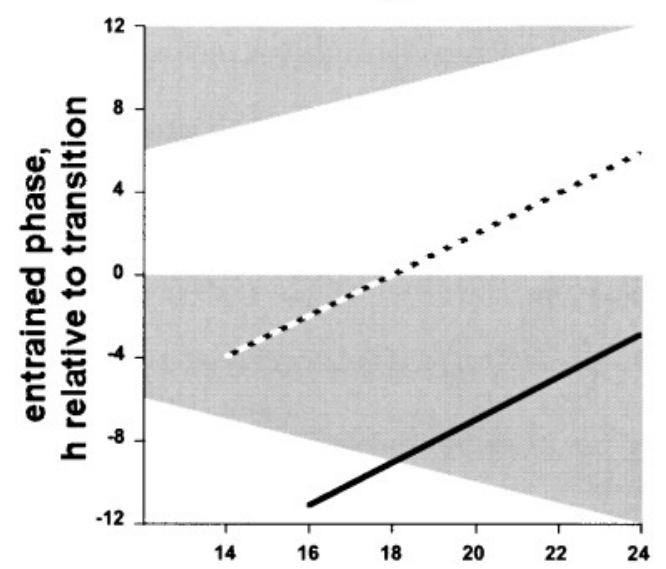

B

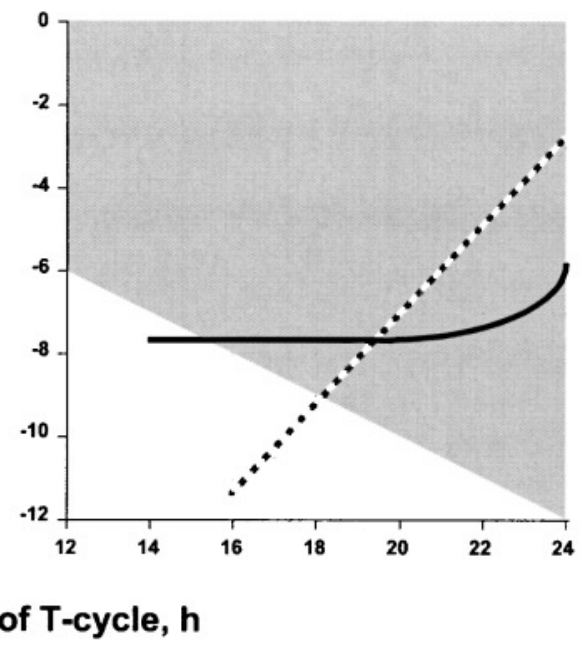

Figure 2. Entrainment characteristics of Neurospora crassa in temperature and light $\mathrm{T}$ cycles. (A) Phases of conidiation in temperature T cycles. The gray area represents the colder $\left(22^{\circ} \mathrm{C}\right)$, the white area the warmer $\left(27^{\circ} \mathrm{C}\right)$ part of each cycle. Daily onsets of conidiation in both $f r q^{+}(-)$and the clock mutant $f r q^{9}$ (---) form parallel lines that are independent of the temperature transitions. (B) Phases of conidiation in light $\mathrm{T}$ cycles $\left(400 \mathrm{nE} \mathrm{m}^{-2} \mathrm{~s}^{-1}\right.$ of cool white fluorescent light and darkness, shown in gray). Unlike the systematic phase changes in temperature cycles, in light cycles the phases are uniformly 7 to $8 \mathrm{~h}$ following lights-off, until the cycle reaches $24 \mathrm{~h}$ (solid line; for comparison, the line for $\mathrm{frq}^{+}$in temperature cycles is replotted from panel A as a dashed line, for which the gray represents the cold incubation). 
With the fundamental characteristics of entrainment described in FRQ-sufficient strains, we investigated entrainment in $f r q^{9}$ (a presumptive functional null mutant) and in $f r q^{10}$ (a knockout) (Aronson et al., 1994a; Loros et al., 1986), both of which have indistinguishable circadian phenotypes. Typically, these mutants do not show a banding rhythm in constant darkness, but there are some conditions in which they do make conidial bands about once per $24 \mathrm{~h}$, namely, in extra-long, $60 \mathrm{~cm}$ race tubes and fairly specific nutritional conditions (Aronson et al., 1994a; Loros and Feldman, 1986). Under these rhythm-permitting conditions, the frq mutation mainly leads to a large variance in $\tau$ but not to a significant change in its mean (Aronson et al., 1994a; Merrow et al., 2001a). The FRQ-less mutants were assayed for entrainment by light and temperature, respectively (Merrow et al., 1999; Roenneberg and Merrow, 2001). In race-tube conditions where a free-running rhythm is not seen in these strains, entrainment with temperature yielded phases that were indicative of an underlying rhythm generator, i.e., the timing of conidial band formation changed relative to the warm-to-cold transition according to the $\mathrm{T}$ [dashed line in Fig. 2(A)].

In contrast to temperature cycles, the null frq mutants failed to synchronize to LD cycles (Merrow et al., 1999), even at fluence rates up to $4 \mu \mathrm{E} \mathrm{m}^{-2} \mathrm{~s}^{-1}$ (1000-fold higher than that required for synchronization of conidiation in the $f r q$-sufficient strain; Merrow and Roenneberg, data not shown). To further understand the blindness of FRQ-less strains, we used a strain where the frq ORF was transformed into the null mutants such that FRQ could be constitutively expressed (Aronson et al., 1994b). Light-regulated conidiation was only restored in these strains when $f r q$ was induced (Merrow et al., 2001b). Thus, the lack of light-regulated conidiation in FRQ-less mutants is indeed due to lack of FRQ and not to background mutations. For synchronization by light, there is apparently no need for direct or indirect light-induction of $f r q$, as has been concluded from experiments with the $\mathrm{frq}^{+}$ strain (Crosthwaite et al., 1995; Froehlich et al., 2002). The transcriptional/translational feedback of frq RNA and protein is also dispensable for this response. These experimental results fully support the hypothesis generated by the model: a FRQ-less oscillator (FLO) remains in frq null mutants. Although important for free-running rhythmicity, the frq-FRQ feedback is dispensable for regulation of spore formation by light, as long as FRQ protein is present.

Experiments combining both light and temperature cycles indicate how multiple zeitgebers work together in the "real world" to "fine tune" entrainment. In high amplitude temperature cycles with light cycles applied in antiphase (i.e., light during the cold phase), light had relatively little effect on the phase of entrainment (Liu et al., 1998). When the amplitude of the temperature cycle was reduced to $5^{\circ} \mathrm{C}$, however, the entrained phase was dominated by the light cycle (Roenneberg and Merrow, 2001).

\section{EXPERIMENTS (2): HOW BLIND IS BLIND?}

Subsequent experiments investigated whether the frq null mutants are completely or selectively blind. An additional light response, i.e., light-induced mycelial carotenoid production, was quantified in $\mathrm{frq}^{+}$and $\mathrm{frq}^{10}$ strains (Merrow et al., 2001b). Both showed robust light-induced carotenoid production — even with similar sensitivities. The only difference was that carotenoid concentrations, at the same, saturating fluences, reached only half the concentration without FRQ compared to the $f r q^{+}$strain. Thus, carotenogene- 
sis and conidiation can either be seen as two distinct light input pathways or two distinct branches off a common light input with FRQ being essential for one but not for the other. Additional support for a separate, or bifurcated light input pathway comes from the very different sensitivities of carotenogenesis and light-regulated conidiation: the latter is 20-fold more sensitive than the former (Merrow et al., 1999; 2001b; Sargent et al., 1956). Because WC-1 is the likely photoreceptor mediating this response (Froehlich et al., 2002; He et al., 2002; Linden et al., 1999) and its levels are regulated by FRQ (Lee et al., 2000; Merrow et al., 2001b), the decreased saturation levels of carotenoids in frq mutant mycelia could be due to the lower level of WC-1 protein (Lee et al., 2000; Merrow et al., 2001b). Investigation of another light-regulated output, gene expression, in the frq null strain confirmed an intact light response, albeit with a decrease in amplitude, again attributable to low WC-1 levels (Arpaia et al., 1993; 1995; Merrow et al., 2001b).

The possibility of a dual light input pathway system suggests a reinvestigation of the hypothesis that WC-1 governs all light responses (He et al., 2002). Blindness of the wc strains for light-regulated conidiation was, so far, only based on release from constant light to constant darkness (Crosthwaite et al., 1997; Russo, 1988). This assay has, however, limited value because under standard race tube conditions $w c$ mutants are arrhythmic in constant darkness, so that no further conidial bands can be measured. When these mutants are submitted to full photoperiod LD cycles, conidiation was entrained (Dragovic et al., 2002), clearly demonstrating that these mutants have a residual light reception, in spite of being completely blind for light-induced mycelial carotenogenesis. Subsequent experiments on knockout mutants (vs. point or RIP mutants, which were first tested) confirm that the entrainment indeed occurs in the complete absence of either WC-1 or WC-2 (Fig. 3). The evidence suggests not only two light-transduction pathways but also two photoreceptors/pigments. The recent annotation of the Neurospora genome contains numerous additional photoreceptor candidates (link to various Neurospora genome projects through http://www.fgsc.net/).

The appearance of light-regulated conidial banding in the $w c$ mutants depends on media composition, with media containing either no carbon source or quinic acid rather than glucose giving the clearest phenotype. Qualitatively, the light-responsive conidial banding in the $w c$ knockout mutants looks similar to those in experiments where FRQ protein is constitutively expressed from an inducible promoter in the frq mutant strains (Merrow et al., 2001). Given that FRQ is unregulated and at low levels in the $w c$ knockout mutants, apparently the abundance of conidiation and the precision of its onset and offset suffer without regulated FRQ levels. This is not trivial, as precision is an important property of circadian systems (Roenneberg and Merrow, 1998; Pittendrigh, 1960).

\section{REVISITING THE MODEL}

Clock-controlled light input pathways appear to be a common feature in many circadian model systems (Bognar et al., 1999; Fleissner and Fleissner, 1987; McWatters et al., 2000). In Neurospora, the convergence of light input components and those involved in the circadian clock suggests a straightforward mechanism for this circadian input regulation. It also shapes how we envision the molecular mechanisms of circadian systems. In plants, light-induction of phytochrome $B(p h y B)$ RNA is gated by the clock (Bognar et al., 1999; McWatters et al., 2000), and, in turn, PHYB has effects on $\tau$ (Somers 

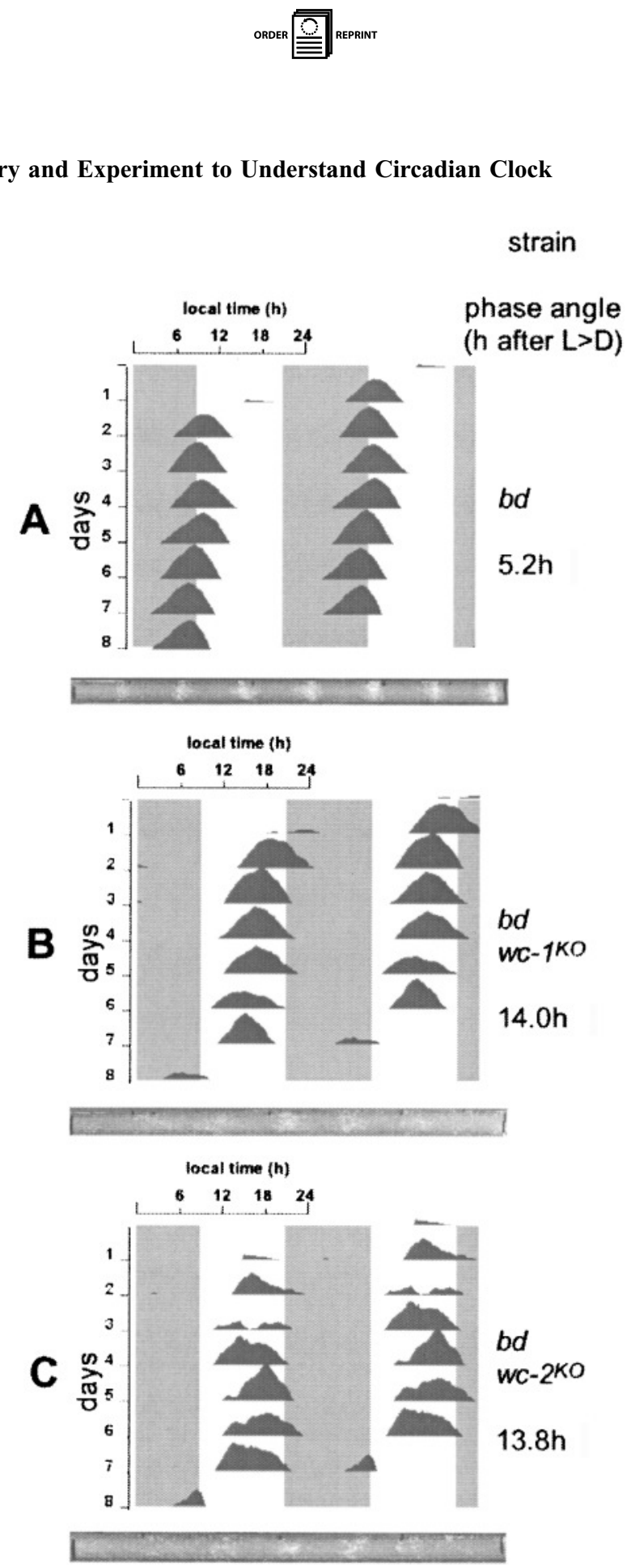

Figure 3. Light-regulated conidiation in the $w c$ mutant strains. Race tubes were incubated in LD $12 \mathrm{~h}: 12 \mathrm{~h}$. Single race tubes are double plotted (gray areas indicate darkness; original scans of the race tubes are shown below each plot). The strain and the delay (in h) between lights-off and conidiation-onset (entrained phase) are given on the right. Indicated phases are the average of six race tubes. The light source was cool white fluorescent light enriched for short wavelengths with solarium light $\left(15 \mu \mathrm{E} \mathrm{m}^{-2} \mathrm{~s}^{-1}\right.$ Osram L36, $5 \mu \mathrm{E} \mathrm{m}^{-2}$ Osram L80). 
et al., 1998). In Neurospora, it is possible to show time-of-day differences in the robustness of light-induced RNA responses (Heintzen et al., 2001; Merrow et al., 2001b) with stronger induction in the subjective night than in the subjective day in the case of the $w c-1$ and al-3 mRNAs. The response correlates with the amount of WC-1 protein found in tissue grown in constant darkness (i.e., more WC-1 during the night: Lee et al., 2000; Merrow et al., 2001b). The word "correlate" should be stressed here, as it is entirely possible that the amplitude of RNA induction by light could also be modulated by other factors.

The combination of modeling and molecular genetic experiments revealed two coupled feedback loops, one of which fulfills the function of a clock-regulated light input (with predictably strong effects on the free-running rhythm) while the other, on its own, still shows rudimentary circadian qualities under entraining conditions. Indeed, evidence beyond the Neurospora clock favors circadian systems as extended networks rather than simple loops. Within the last year, two new, interlocking feedback loops were added to the ones already known for the mammalian system (Honma et al., 2002; Preitner et al., 2002). When these numerous negative feedback loops are treated as individual components within a complex network (Roenneberg and Merrow, 2003), it becomes apparent that the circadian molecular system is difficult to explain based on straightforward genetic analyses.

Figure 4 diagrams the Neurospora circadian system similarly to how we have recently drawn the circadian network in mammals (Roenneberg and Merrow, 2003). As for the known clock components, FRQ, WC-1, WC-2, and VVD, only one, FRQ, has an

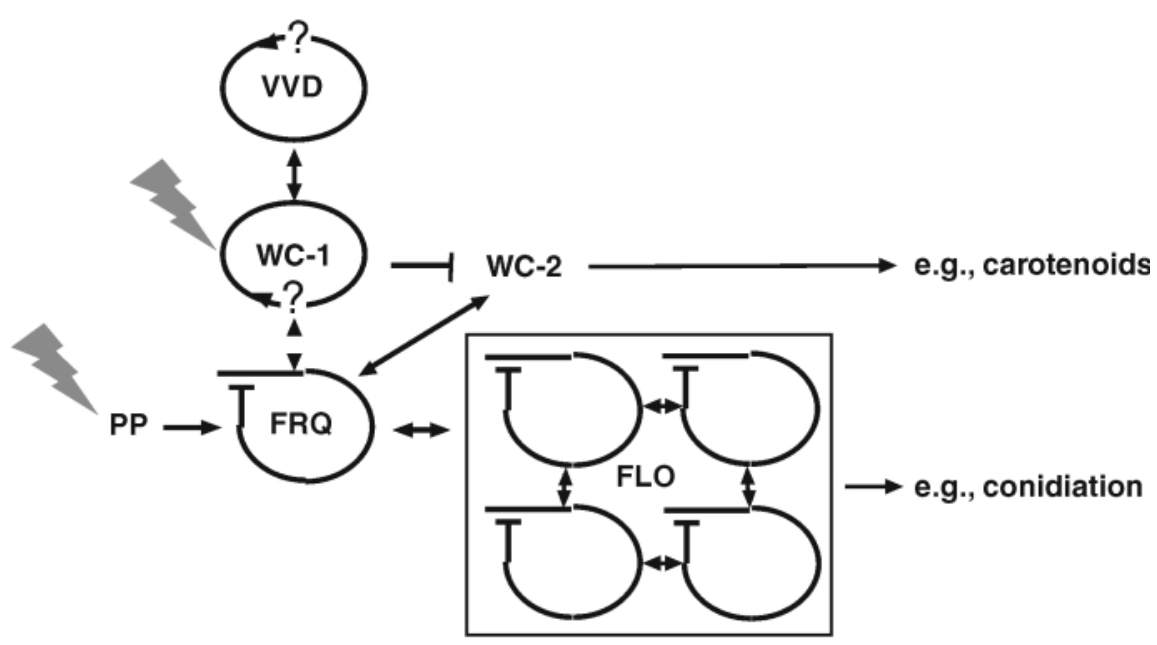

Figure 4. A schematic representation of the Neurospora molecular circadian network. The wc-1, $w c-2, f r q$, and $v v d$ gene products are known to impact the endogenous daily timing system. They all regulate each other, either directly or indirectly. The FRQ-less oscillator (FLO), which generates freerunning circadian rhythms under special conditions (Aronson et al., 1994; Loros et al., 1986) and is entrained by temperature cycles (Merrow et al., 1999), could add further feedback loops, thus forming an extensive feedback network with specific domains serving different functions. The lightning bolts indicate light inputs and "PP" indicates as yet unknown photoreceptor/ photopigment(s). 
individual, self-regulated feedback loop. It inhibits its own transcriptional activation (Aronson et al., 1994b; Froehlich et al., 2002), and thus, in theory, given constitutive expression of its regulators, could achieve an oscillation. WC-1 protein, but not RNA, is rhythmically expressed in (DD) (Lee et al., 2000; Merrow et al., 2001b), although RNA levels can be modulated by FRQ (Merrow et al., 2001b), and a self-sustained circadian rhythm is rescued in $w c-1$ mutants with constitutive overexpression of WC-1 (Cheng et al., 2001). Thus, WC-1 is not placed in a feedback loop per se although its regulation is probably important for the circadian system. Constitutive expression of WC-2 also supports a free-running rhythm, and neither $w c-2$ RNA nor protein oscillate, so it serves as a parameter of the system. VIVID has negative effects on WC-1, and oscillates for one cycle in DD (Heintzen et al., 2001). It is not light-induced in the $w c-1$ knockout strain, indicating regulation by WC-1 (Lee et al., 2003). The four feedback loops in the FLO are conjectured, and are representative of some of the many feedbacks embedded in the cell's biochemistry that must talk to one another.

The diagram is not only an alternative description of the molecular circadian machinery but also suggests distinct domains within the network: several individual feedbacks separately receive direct or indirect light input, and others participate in the generation of circadian rhythmicity that remains entrainable by temperature cycles in the absence of the light input $\operatorname{loop}(\mathrm{s})$. Another benefit of conceptualizing the network as individual feedbacks is that it highlights likely locations for new components, e.g., the transcription factors that regulate basal expression levels of $w c-1, w c-2, f r q$, or those involved in rhythmic conidiation in temperature cycles in FRQ-less strains (the FRQ-less oscillator, FLO).

Several experimental results support the fundamental role of a feedback network as a general basis of circadian rhythmicity. Recent experimental results show that the freerunning rhythmicity of some clock mutant mice is conditional, e.g., some have poorly or nonsustained circadian rhythms in constant darkness but show self-sustained rhythmic activity in constant light (Steinlechner et al., 2002; Spoelstra et al., 2002). The network concept also predicts that the elimination of one loop may disturb the network, so that its output becomes arhythmic, while the elimination of an additional loop may reestablish rhythmicity. Recent experiments with mammalian double clock mutants show evidence for this possibility (Oster et al., 2002).

The network view of the molecular circadian system is also supported by modeling the evolution of the circadian clock with a set of simple, coupled feedback loops (Roenneberg and Merrow, 2002). Although circadian research has shown the central role of transcriptional/translational autoregulation, a network of feedbacks must have already existed at all levels of regulation in primitive, clockless cells. Feedbacks are a common control mechanism in metabolic pathways (see Roenneberg and Merrow, 1998), albeit with periods much shorter than $24 \mathrm{~h}$. Well-defined, transcriptional/translational feedbacks can have self-sustained periods much shorter than 24h (Hirata et al., 2002; Hoffmann et al., 2002; Elowitz and Leibler, 2000). The basic cellular biochemistry consists of a network of coupled feedback loops, including many potential, short-period oscillators. When such a network is modeled and is "stimulated" by rhythmic inputs (similar to a $24 \mathrm{~h}$ zeitgeber), the network's responses are chaotic, although each individual, isolated loop can be readily driven by the "zeitgeber signal." Thus, an additional mechanism must have allowed complex cellular feedback networks to respond in an organized manner to light and darkness or warm and cold, even before a full fledged circadian system existed. 
A solution to this problem is to add a zeitnehmer (German for "time taker") function, a feedback from the output of the network onto its input pathway (Roenneberg and Merrow, 1998; 2002). Although zeitnehmer functions allow the system to respond adequately to rhythmic stimulation, the system is still not self-sustained with a circadian period under constant conditions. The evolution of a circadian system based on such a feedback network is, however, simulated in the model by changing the coupling strength between the individual feedback loops.

The implications of such a model are several. Many of the genes participating in this network may well be genes that are essential for survival, thus they would be difficult to find in mutant screens. On the other hand, if the model is correct, nonclock characteristics can be used to identify clock gene candidates that would not be found by traditional methods. For example, a mutant screen for loss of an organized response to temperature cycles could be performed either in organisms with no apparent clock (such as yeast) or in mutants with a disrupted clock.

\section{CONCLUSION}

Studying the circadian system with a forward genetics approach has resulted in the description of a transcription/translation feedback loop as central to the clock mechanism in all model systems. Based on their strong impact on circadian rhythms, these clock components have come to be recognized as clock genes, or "core clock component."

The exercise of assigning circadian function to a given gene or molecule has an intellectual lineage. The first generation would be the "one-gene-one-enzyme hypothesis," as was originally described using Neurospora (Beadle, 1945). The second generation (the molecular genetic revolution) produced the transcription/translation feedback loop, faithfully propagating the idea that the phenotype in a mutant is a direct readout of the mutation. Because circadian research has focused its efforts (using similar genetic approaches) on those genes and molecules that have profound effects on circadian rhythms, it may have tilted towards transcriptional regulation as a basis for circadian rhythmicity. The discovery that there are clearly additional domains of circadian regulation, as described here, and by work on Neurospora in other labs (Lakin-Thomas, 2000; Lakin-Thomas and Brody, 2000) may be the beginning of the third generation.

The detailed kinetic description of the known clock components and the continuing discovery of new clock components reveal an extensive coregulation. Quantitative genetic analyses have shown, for example, unexpected interactions among the components of the olfactory pathway in Drosophila (thought to represent epistatic effects (Federowicz et al., 1998)). It could be that sensory transduction pathways commonly feature interdependent regulation, and any parsimonious interpretation of genetic results would become misleading. Has the Neurospora clock evolved from two light input pathways that feed back onto each other with the consequence of circadian, self-sustained rhythmicity or has a circadian molecular network evolved to improve the processing of light information?

Nonstraight-forward genetic interactions among clock mutations have already been demonstrated in Neurospora (Lakin-Thomas and Brody, 1981; Morgan et al., 2001). This cellular system, for which uniform tissue types can easily be manipulated and analyzed, appears especially suited to study coregulatory effects. This task is a particularly difficult challenge in mice, where the circadian system of the whole animal is clearly a composite 
of numerous cellular and organ clocks (Herzog and Tossini, 2001), and dissection of discrete genetic effects is confounded by their interactions. With the Neurospora genome annotation available, and forward and reverse genetic tools online, the use of novel mutant screens together with physiological experimentation should generate a panel of novel clock genes. The only mutant screens that have been used in Neurospora to date are those under constant conditions, but entrainment, as it controls appropriate temporal expression of function, is surely one of the most important circadian properties. We have started screening for mutants under entrained conditions (light or temperature cycles, both $24 \mathrm{~h}$ and non-24h T). The first results indicate that there are many clock genes that impact the phase of entrainment in this fungus (M. Mason and K. Sveric, unpublished). Given the parallels between the clock mechanism in Neurospora and those of all other model systems, these novel clock genes should be relevant for identification of similar components in other circadian model systems.

\section{ACKNOWLEDGMENTS}

We thank Vera Schiewe, Astrid Bauer and Casper Roenneberg for their help and support. This was financially supported by the DFG, the Friedrich-Bauer- and MeyerStruckmann-Stiftungs and the Eppendorf Company, Hamburg.

\section{REFERENCES}

Aronson, B. D., Johnson, K. A., Dunlap, J. C. (1994a). The circadian clock locus frequency: a single ORF defines period length and temperature compensation. Proc. Natl. Acad. Sci. USA 91:7683-7687.

Aronson, B. D., Johnson, K. A., Loros, J. J., Dunlap, J. C. (1994b). Negative feedback defining a circadian clock: autoregulation of the clock gene frequency. Science 263:1578-1584.

Arpaia, G., Loros, J. J., Dunlap, J. C., Morelli, G., Macino, G. (1993). The interplay of light and the circadian clock. Plant Physiol. 102:1299-1305.

Arpaia, G., Loros, J. J., Dunlap, J. C., Morelli, G., Macino, G. (1995). Light induction of the clock-controlled gene $c c g-1$ is not transduced through the circadian clock in Neurospora crassa. Mol. Gen. Genet. 247:157-163.

Aschoff, J. (1979). Circadian rhythms: general features and endocrinological aspects. In: Krieger, D., ed. Endocrine Rhythms. New York: Raven Press, pp. 1-60.

Aschoff, J., Pohl, H. (1978). Phase relations between a circadian rhythm and its zeitgeber within the range of entrainment. Naturwiss. 65:80-84.

Ballario, P., Vittorioso, P., Magrelli, A., Talora, C., Cabibbo, A., Macino, G. (1996). White collar-1, a central regulator of blue light responses in Neurospora, is a zinc finger protein. EMBO J. 15(7):1650-1657.

Beadle, G. W. (1945). Biochemical genetics. Chem. Revs. 37:15-96.

Bognar, L. K., Adam, A. H., Thain, S. C., Nagy, F., Millar, A. J. (1999). The circadian clock controls the expression pattern of the circadian input photoreceptor, phytochrome B. Proc. Natl. Acad. Sci. USA 96:14652-14657.

Bruce, V. (1960). Environmental entrainment of circadian rhythms. Cold Spring Harbor Symp. Quant. Biol. 25:29-48. 
Buskey, E., Mills, L. (1983). The effects of dinoflagellate bioluminescence on the swimming behavior of a marine copepod. Limnol. Oceanog. 28(zooplankton ecology):575-579.

Cheng, P., Yang, Y., Liu, Y. (2001). Interlocked feedback loops contribute to the robustness of the Neurospora circadian clock. Proc. Natl. Acad. Sci. USA 98:7408-7413.

Cheng, P., Yang, Y., Wang, L., He, Q., Liu, Y. (2002). WHITE COLLAR-1, a multifunctional Neurospora protein involved in the circadian feedback loops, light sensing, and transcription repression of wc-2. J. Biol. Chem. 278(6):3801-3808.

Crosthwaite, S. K., Loros, J. J., Dunlap, J. C. (1995). Light-induced resetting of a circadian clock is mediated by a rapid increase in frequency transcript. Cell 81:1003-1012.

Crosthwaite, S. K., Dunlap, J. C., Loros, J. J. (1997). Neurospora wc-1 and wc-2: transcription, photoresponses, and the origin of circadian rhythmicity. Science 276:763-769.

Dragovic, Z., Tan, Y., Görl, M., Roenneberg, T., Merrow, M. (2002). Light reception and circadian behavior in 'blind' and 'clock-less' mutants of Neurospora crassa. EMBO J. 21(14):3643-3651.

Elowitz, M. B., Leibler, S. (2000). A synthetic oscillatory network of transcriptional regulators. Nature 403:335-338.

Federowicz, G. M., Fry, J. D., Anholt, R. R., Mackay, T. F. (1998). Epistatic interactions between smell-impaired loci in Drosophila melanogaster. Genetics 148:1885-1891.

Feldman, J. F., Hoyle, M. N. (1973). Isolation of circadian clock mutants of Neurospora crassa. Genetics 75:605-613.

Fleissner, G., Fleissner, G. (1987). Efferent control of visual sensitivity in arthropod eyes: with emphasis on circadian rhythms. In: Lindauer, M., ed. Information Processing in Animals. Vol. 5. Stuttgart, New York: Gustav Fischer.

Fleissner, G., Fleissner, G. (1996). The scorpion's clock as a model for feedback mechanisms in circadian systems. In: Brownell, P., Polis, G., eds. Scorpion Biology and Research. Oxford: Oxford University Press, pp. 11-17.

Froehlich, A. C., Liu, Y., Loros, J. J., Dunlap, J. C. (2002). White Collar-1, a circadian blue light photoreceptor, binding to the frequency promoter. Science 297:815-819.

Glass, L., Mackey, M. C. eds. (1988). From clocks to chaos. From Clocks to Chaos. Princeton Univ. Press.

Görl, M., Merrow, M., Huttner, B., Johnson, J., Roenneberg, T., Brunner, M. (2001). A PEST-like element in FREQUENCY determines the length of the circadian period in Neurospora crassa. EMBO J. 20(24):7074-7084.

Hardin, P. E., Hall, J. C., Rosbash, M. (1990). Feedback of the Drosophila period gene product on circadian cycling of its messenger RNA levels. Nature 343:536-540.

He, Q., Cheng, P., Yang, Y., Wang, L., Gardner, K. H., Liu, Y. (2002). White Collar-1, a DNA binding transcription factor and light sensor. Science 297:840-843.

Heintzen, C., Loros, J. J., Dunlap, J. C. (2001). The PAS protein VIVID defines a clockassociated feedback loop that represses light input, modulates gating, and regulates clock resetting. Cell 104:453-464.

Herzog, E. D., Tossini, G. (2001). The mammalian circadian clock shop. Semin. Cell Dev. Biol. 12(4):295-304.

Hirata, H., Yoshiura, S., Ohtsuka, T., Bessho, Y., Harada, T., Voshikawa, K., Kageyama, R. (2002). Oscillatory expression of the bHLH factor Hes1 regulated by a negative feedback loop. Science 298:840-843. 


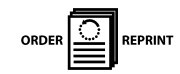

Combining Theory and Experiment to Understand Circadian Clock

Hoffmann, A., Levchenko, A., Scott, M. L., Baltimore, D. (2002). The I $\kappa$ B-NF- $\kappa$ B signaling module: temporal control and selective gene activation. Science 298:1241-1245.

Holst, E. V. (1939). Die relative Koordination als Phänomen und als Methode zentralnervöser Funktionsanalyse. Ergebn. Physiol. 42:228-306.

Honma, S., Kawamoto, T., Takagi, Y., Fujimoto, K., Sato, F., Noshiro, M., Kato, V., Honma, K.-I. (2002). Dec1 and Dec2 are regulators of the mammalian clock. Nature 419:841-844.

Kramer, C., Loros, J. J., Dunlap, J. C., Crosthwaite, S. K. (2003). Role for antisense RNA in regulating circadian clock function in Neurospora crassa. Nature 421:948-952.

Lakin-Thomas, P. L. (2000). Circadian rhythms: new functions for old clock genes? TIGs 16(3):135-142.

Lakin-Thomas, P. L., Brody, S. (1981). Circadian rhythms in Neurospora crassa; additive and non-additive gene interactions. J. Cell Biol. 91:20a.

Lakin-Thomas, P. L., Brody, S. (2000). Circadian rhythms in Neurospora crassa: lipid deficiencies restore robust rhythmicity to null frequency and white-collar mutants. Proc. Natl. Acad. Sci. USA 97:256-261.

Lee, D.-H., Mittag, M., Sczekan, S., Morse, D., Hastings, J. W. (1993). Molecular cloning and genomic organization of a gene for luciferin-binding protein from the dinoflagellate Gonyaulax polyedra. J. Biol. Chem. 268:8842-8850.

Lee, K., Loros, J. J., Dunlap, J. C. (2000). Interconnected feedback loops in the Neurospora circadian system. Science 289:107-110.

Lee, K., Dunlap, J. C., Loros, J. J. (2003). Roles for WHITE COLLAR-1 in circadian and general photoreception in Neurospora crassa. Genetics 168:103-114.

Linden, H., Ballario, P., Arpaia, G., Macino, G. (1999). Seeing the light: news in Neurospora blue light signal transduction. Adv. Genet. 41:35-54.

Liu, Y., Merrow, M., Loros, J. L., Dunlap, J. C. (1998). How temperature changes reset a circadian oscillator. Science 281:825-829.

Liu, Y., Loros, J., Dunlap, J. C. (2000). Phosphorylation of the Neurospora clock protein FREQUENCY determines its degradation rate and strongly influences the period length of the circadian clock. Proc. Natl. Acad. Sci. USA 97:234-239.

Loros, J. J., Feldman, J. F. (1986). Loss of temperature compensation of circadian period length in the frq-9 mutant of Neurospora crassa. J. Biol. Rhythms 1:187-198.

Loros, J. J., Richman, A., Feldman, J. F. (1986). A recessive circadian clock mutation at the frq locus of Neurospora crassa. Genetics 114:1095-1110.

McClung, C. R., Fox, B. A., Dunlap, J. C. (1989). The Neurospora clock gene frequency shares a sequence element with the Drosophila clock gene period. Nature 339:558-562.

McWatters, H. G., Bastow, R. M., Hall, A., Millar, A. J. (2000). The ELF3 zeitnehmer regulates light signalling to the circadian clock. Nature 408:716-720.

Merrow, M., Brunner, M., Roenneberg, T. (1999). Assignment of circadian function for the Neurospora clock gene frequency. Nature 399:584-586.

Merrow, M., Roenneberg, T., Macino, G., Franchi, L. (2001a). A fungus among us: the Neurospora crassa circadian system. Semin. Cell Dev. Biol. 12(4):279-285.

Merrow, M., Franchi, L., Dragovic, Z., Görl, M., Johnson, J., Brunner, M., Macino, G., Roenneberg, T. (2001b). Circadian regulation of the light input pathway in Neurospora crassa. EMBO J. 20(3):307-315. 
Morgan, L. W., Feldman, J. F., Bell-Pedersen, D. (2001). Genetic interactions between clock mutations in Neurospora crassa: can they help us to understand complexity. Phil. Trans. Royal Soc. Lond. 356:1717-1724.

Morse, D., Hastings, J. W., Roenneberg, T. (1994). Different phase responses of two circadian oscillators in Gonyaulax. J. Biol. Rhythms 9:263-274.

Oster, H., Yasui, A., van der Horst, G. T. J., Albrecht, U. (2002). Disruption of $m C r y 2$ restores circadian rhythmicity in $m P e r 2$ mutant mice. Genes Dev. 16:2633-2638.

Pittendrigh, C. S. (1960). Circadian rhythms and the circadian organization of living systems. Cold Spring Harbor Symp. Quant. Biol., 25:159-184.

Preitner, N., Damiola, F., Lopez-Molina, L., Zakany, J., Duboule, D., Albrecht, U., Schibler, U. (2002). The orphan nuclear receptor REV-ERB $\alpha$ controls circadian transcription within the positive limb of the mammalian circadian oscillator. Cell 110(2):251-260.

Rensing, L., Mohsenzadeh, S., Ruoff, P., Meyer, U. (1997). Temperature compensation of the circadian period length - a special case among general homeostatic mechanism of gene expression. Chronobiol. Int. 14(5):481-498.

Roenneberg, T., Hastings, J. W. (1988). Two photoreceptors influence the circadian clock of a unicellular alga. Naturwissenschaften 75:206-207.

Roenneberg, T., Morse, D. (1993). Two circadian oscillators in one cell. Nature 362:362-364.

Roenneberg, T., Rehman, J. (1996). Nitrate, a nonphotic signal for the circadian system. FASEB J. 10:1443-1447.

Roenneberg, T., Deng, T.-S. (1997). Photobiology of the Gonyaulax circadian system: I. Different phase response curves for red and blue light. Planta 202:494-501.

Roenneberg, T., Merrow, M. (1998). Molecular circadian oscillators-an alternative hypothesis. J. Biol. Rhythms 73:167-179.

Roenneberg, T., Merrow, M. (2001). The role of feedbacks in circadian systems. In: Honma, K., Honma, S., eds. Zeitgebers, Entrainment and Masking of the Circadian System. Sapporo: Hokkaido Univ. Press, pp. 113-129.

Roenneberg, T., Merrow, M. (2002). Life before the clock-modeling circadian evolution. J. Biol. Rhythms 17(6):495-505.

Roenneberg, T., Merrow, M. (2003). The network of time: understanding the molecular circadian system. Curr. Biol. 13:R198-R207.

Roenneberg, T., Daan, S., Merrow, M. (2003). The art of entrainment. J. Biol. Rhythms, in press.

Ruoff, P., Rensing, L. (1996). The temperature-compensated Goodwin model simulates many circadian clock properties. J. Theor. Biol. 179:275-285.

Ruoff, P., Mohsenzadeh, S., Rensing, L. (1996). Circadian rhythms and protein turnover: the effect of temperature on the period lengths of clock mutants simulated by the Goodwin oscillator. Naturwiss. 83(11):514-517.

Ruoff, P., Rensing, L., Kommedal, R., Mohsenzadeh, S. (1997). Modelling temperature compensation in chemical and biological oscillators. Chronobiol. Int. 14(5):499-510.

Russo, V. E. (1988). Blue light induces circadian rhythms in the $b d$, mutant of Neurospora: double mutants $b d, w c-1$ and $b d, w c-2$ are blind. Photochem. Photobiol. 2(1):59-65. 


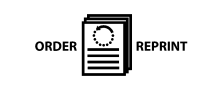

Combining Theory and Experiment to Understand Circadian Clock

Sargent, M. L., Briggs, W. R., Woodward, D. O. (1956). Circadian nature of a rhythm expressed by an invertaseless strain of Neurospora crassa. Plant Physiol. 41:1343-1349.

Schwerdtfeger, C., Linden, H. (2000). Localization and light-dependent phosphorylation of White collar 1 and 2, the two central components of blue light signalling in Neurospora crassa. Eur. J. Biochem. 267:414-422.

Shrode, L. B., Lewis, Z. A., White, L. D., Bell-Pedersen, D., Ebbole, D. J. (2001). vvd is required for light adaptation of conidiation-specific genes of Neurospora crassa but not circadian conidiation. Fung. Gen. Biol. 32(3):169-181.

Somers, D. E., Devlin, P. F., Kay, S. A. (1998). Phytochromes and cryptochromes in the entrainment of the Arabidopsis circadian clock. Science 282:1488-1490.

Spoelstra, K., Okeljewicz, M., Daan, S. (2002). Restoration of self-sustained rhythmicity by the mutant clock allele in mice in constant illumination. J. Biol. Rhythms 17(6):520-525.

Steinlechner, S., Jacobmeier, B., Scherbarth, F., Dembach, H., Kruse, F., Albrecht, U. (2002). Robust circadian rhythmicity of Perl and Per 2 mutant mice in constant light and dynamics of Perl and Per2 gene expression under long and short photoperiods. J. Biol. Rhythms 17:202-209.

Talora, C., Franchi, L., Linden, H., Ballario, P., Macino, G. (1999). Role of a white collar1-white collar-2 complex in blue-light signal transduction. EMBO J. 18:4961-4068.

Yang, Y., Cheng, P., Liu, Y. (2002). Regulation of the Neurospora circadian clock by casein kinase II. Genes Dev. 16:994-1006. 